\title{
Enabling Equal Access to Molecular Diagnostics: What Are the Implications for Policy and Health Technology Assessment?
}

\author{
Juliette Plun-Favreau ${ }^{\mathrm{a}}$ Kaisa Immonen-Charalambous ${ }^{\mathrm{b}}$ Lotte Steuten $^{\mathrm{e}}$ \\ Anja Strootker ${ }^{c}$ Roman Rouzier ${ }^{f}$ Denis Horgan ${ }^{d}$ Mark Lawler $^{g}$ \\ ${ }^{a}$ Genomic Health International SARL, Geneva, Switzerland; ${ }^{b}$ European Patients' Forum, 'Incite Public Affairs, and \\ ${ }^{d}$ European Alliance for Personalised Medicine, Brussels, Belgium; ${ }^{e}$ Fred Hutchinson Cancer Researcher Center, \\ Panaxea B.V., Seattle, Wash., USA; ${ }^{\mathrm{f} I n s t i t u t}$ Curie, Paris, France; ${ }^{9}$ Centre for Cancer Research and Cell Biology, \\ Queen's University Belfast, Belfast, UK
}

\section{Key Words}

Europe $\cdot$ Health technology assessment $\cdot$ Molecular diagnostics Patient access $\cdot$ Personalised medicine .

Policy · Reimbursement

\begin{abstract}
Molecular diagnostics can offer important benefits to patients and are a key enabler of the integration of personalised medicine into health care systems. However, despite their promise, few molecular diagnostics are embedded into clinical practice (especially in Europe) and access to these technologies remains unequal across countries and sometimes even within individual countries. If research translation and the regulatory environments have proven to be more challenging than expected, reimbursement and value assessment remain the main barriers to providing patients with equal access to molecular diagnostics. Unclear or non-existent reimbursement pathways, together with the lack of clear evidence requirements, have led to significant delays in the assessment of molecular diagnostics technologies in certain countries. Additionally, the lack of dedicated diagnostics budgets and the siloed nature of resource allocation within certain health care systems have significantly delayed diagnostics commissioning. This article will con-
\end{abstract}

\begin{tabular}{ll}
\hline KARGER & $\begin{array}{l}\text { () } 2016 \text { The Author(s) } \\
\text { Published by S. Karger AG, Basel }\end{array}$ \\
& 1662-4246/16/0193-0144\$39.50/0 \\
E-Mail karger@karger.com & This article is licensed under the Creative Commons Attribution- \\
www.karger.com/phg & NonCommercial-NoDerivatives 4.0 International License (CC BY- \\
& NC-ND) (http://www.karger.com/Services/OpenAccessLicense). \\
& Usage and distribution for commercial purposes as well as any dis- \\
& tribution of modied material requires written permission
\end{tabular}

sider the perspectives of different stakeholders (patients, health care payers, health care professionals, and manufacturers) on the provision of a research-enabled, patientfocused molecular diagnostics platform that supports optimal patient care. Through the discussion of specific case studies, and building on the experience from countries that have successfully integrated molecular diagnostics into clinical practice, this article will discuss the necessary evolutions in policy and health technology assessment to ensure that patients can have equal access to appropriate molecular diagnostics.

(c) 2016 The Author(s) Published by S. Karger AG, Basel

\section{Introduction}

The recently adopted EU Council Conclusions on Personalised Medicine for Patients [1] refer to personalised medicine as 'a medical model using characterisation of individuals' phenotypes and genotypes (e.g. molecular profiling, medical imaging, lifestyle data) for tailoring the right therapeutic strategy for the right person at the right time, and/or to determine the predisposition to disease and/or to deliver timely and targeted prevention'. In this respect, personalised medicine has the potential to opti- 
mise the timely delivery and dosing of treatments so that patients can receive the most benefit for the least amount of risk and harm, minimising both the unnecessary side effects of potentially toxic treatments such as chemotherapy and the delays associated with the 'trial-and-error' process that many patients endure to obtain the correct diagnosis and treatment for their condition [2].

Molecular diagnostic testing plays a key role in personalised medicine, as it is embedded at every stage of the treatment pathway - from providing reliable information that informs the optimal treatment for the individual patient to precise monitoring of its effectiveness. Molecular diagnostics identify particular biological traits - often within the genome of the disease tissue of individuals that can underpin diagnoses, prognoses, or predictions of disease recurrence. Molecular technologies can be enablers of a personalised medicine care plan, as they help classify disease subtypes and identify patients most likely to respond to a particular therapeutic or preventative modality while sparing those who will not benefit from side effects and potential costs [3].

Despite their widely recognised promise, the use of molecular diagnostics in clinical practice across Europe is still limited. Health care systems in Europe are not designed to support personalised medicine approaches and related innovative diagnostic technologies [4]. This is reflected in a lack of appropriate patient access and clear pathways for reimbursing molecular diagnostics. Although the results of molecular diagnostic tests can help influence therapy selection, reimbursement of these tests is limited. New pricing and reimbursement models which reward innovation and value and are transparent are urgently needed in order to ensure that patients can benefit from the results of these tests and receive equitable care leading to improved clinical outcomes [3].

\section{Assessing the Value of Molecular Diagnostics in Europe}

\section{Benefits and Challenges to Patients, Physicians, and Payers}

Molecular diagnostics offer important benefits to patients, physicians, and payers, because they can provide patients with the use of safer and more effective therapies as well as increased confidence about treatment decisions. For example, KRAS mutation testing can support patients with metastatic colorectal cancer (CRC) and their physicians in making informed decisions about effective treatment [5].

Equal Access to Molecular Diagnostics
However, in order to allow patients to fully benefit from molecular diagnostics, some important concerns need to be addressed, such as the need for patients in Europe to have equal access to these new technologies. In addition, clear communication about the information provided by these new technologies is key in order to allow a patient to make informed decisions about his/her treatment. This information must be patient adapted, and patients must be involved in the preparation of appropriate print and online materials.

From a physician's viewpoint, molecular diagnostics can provide physicians with information to assess risks and make the best treatment decisions for individual patients. Additional benefits perceived by physicians are the potential reduction of medical errors through personalised approaches and potentially a reduction of treatment costs [6]. A concern to physicians involves personalised medicine being seen as too disruptive, since it often leads to changes in current clinical practice (e.g. change in prescription behaviour). In order to incorporate the benefits into daily practice and patient care, and to address health care professionals' concerns, it is key that targeted education programmes for health care professionals are developed and implemented [7].

By personalising and optimising treatment, the use of molecular diagnostics brings the promise of more costeffective health care, supporting sustainable health care systems. Access to personalised medicine and related new diagnostic technologies can help eliminate many inefficiencies, such as trial-and-error dosing, the potential for increased hospitalisation time due to adverse drug reactions, and the problem of late diagnoses. It may also enhance the effectiveness of therapies through better-tailored treatment administration.

However, personalised medicine also raises concerns and questions from payers. In a context of health care budget restrictions, payers are anxious about any budget impact that these new technologies may have. In particular, they ask that cost-effectiveness be demonstrated and are concerned that new targeted therapies - because they address a smaller population - may ultimately become more expensive. Finally, in most countries, health technology assessment (HTA) bodies currently lack the capacity to address this new complexity, and clear reimbursement pathways to allow timely and equitable access for patients have not yet been established. Payers' and HTA agencies' considerations about personalised medicine have been described in detail in a recent publication from the International Society for Pharmacoeconomics and Outcomes Research (ISPOR) Working Group on 
Personalised Medicine [8]. Given the important benefits from molecular diagnostics, including their cost-effectiveness, the challenges faced by payers and HTA agencies need to be addressed with reliable and robust evidence.

\section{A Need for Clear and Efficient Reimbursement Pathways}

An important cause of limited access to and use of molecular diagnostics across Europe is a lack of clear reimbursement pathways. Pricing and reimbursement decisions are made at the country level, and this seems unlikely to change in the near future. In many countries, reimbursement pathways are either lacking or are not clearly defined, for instance in Germany (see Box 1). Where reimbursement pathways exist, they usually involve complex and time-consuming processes, for instance in France (see Box 2).

\section{Box 1}

In Germany, the health care system is split into two sectors (the outpatient and the inpatient sector) involving separate procedures and stakeholders [9]. In the inpatient sector it is expected that hospitals fund the new diagnostic test from their existing budget until a new code and a new tariff are established. Because this process can take at least 3 years, it significantly limits the introduction of new diagnostics, especially if the price of a diagnostic is relatively high. An innovation funding pathway exists (NUB, i.e. Neue Untersuchungs- und Behandlungsmethoden); however, it is rarely granted to new diagnostic tests and rather seems to be reserved for innovative medicines and medical devices. In the outpatient sector, the pathway to reimbursement is not entirely clear for diagnostics. Indeed, the German HTA body (IQWIG) can be charged by the Federal Joint Committee (GBA) to start a new assessment. This, however, usually requires a high demand from physicians and patients and therefore does not happen as soon as the new diagnostic test is made available. Finally, for diagnostics that are employed in both sectors, the situation becomes even more complicated and sometimes ends in actual system failure.

\section{Box 2}

In France, manufacturers cannot request reimbursement for their diagnostic tests. Reimbursement for such technologies must be requested by a scientific society. Once the reimbursement dossier is submitted by the scientific society/societies, it needs to be prioritised by the HAS (Haute Autorité de Santé) for an HTA. Once the assessment is completed, pricing and coding discussions may take place between the national payer (Union Nationale des Caisses d'Assurance Maladie) [10] and the Physicians Associations that are intending to use the diagnostic test. Overall, the process is quite long and not governed by specific timelines. This has implications for small diagnostic manufacturers, as many of them may not be able to sustain such a long process financially.
There is an urgent need to clarify and streamline national reimbursement pathways and processes, but also to ensure that the perspective of key stakeholders is being fully considered in those discussions.

\section{A Need for a Dedicated HTA Framework}

In order to truly realise the potential of these new diagnostic technologies in Europe, HTA processes need to be adapted. In most European countries, HTAs are used to inform funding and reimbursement decisions. Without developing an understanding of citizens' concerns and expectations, HTA agencies and decision-makers may miss major drivers and barriers to the successful integration of personalised medicine into routine care. Unfortunately, current HTAs are rarely concerned with assessing the wider societal benefits and risks of health technologies and services. Without recognising these wider aspects of the proposition of a societal value of personalised medicine, suboptimal incentivisation of corresponding innovation activity is a likely consequence [3]. A new and broader definition of value, which explicitly includes the full benefits from personalised medicine and molecular diagnostics, needs to be developed and integrated into HTA, pricing, and reimbursement systems [11-13]. In this respect, patients' involvement in HTA and reimbursement is key [14], as described in the related section below.

In addition to this, there needs to be a clear framework defining the assessment of molecular diagnostics. Evidence requirements and criteria for decision-making of HTA bodies should be clearly established. HTA processes would benefit from being tailored specifically to the ways in which diagnostics are developed and tested. In particular, there should be specific requirements for evidence generation. First, the evidence level grid should be made specific to the development of diagnostics, as such technologies are developed using different methodologies and they have shorter cycles of development, which needs to be taken into account to allow health care systems to keep pace with innovation. Secondly, while traditional cost-effectiveness analysis conducted as part of HTA focuses on three key elements - i.e. (1) life years gained, (2) improvements in patient quality of life, and (3) cost savings within the health care system - these need to be reevaluated to take into account other dimensions of value that are specific to the molecular diagnostics pathway, such as increased certainty in treatment decision-making, the so-called value of knowing [11-13]. This issue, however, requires further research before it can be accurately captured, and it is being discussed elsewhere [15]. Finally,
Plun-Favreau et al. 
the implementation of HTA guidance is key. In the UK, for example, the implementation of National Institute for Health and Clinical Excellence (NICE) guidance is limited by the fact that it is not legally binding for diagnostics [16]. Indeed, unlike with drugs, there is no legal obligation for the National Health Service to fund new diagnostics recommended by NICE. This obviously significantly limits the implementation of NICE guidance when it is related to diagnostics. There is also no monitoring in place to follow up on the implementation of this guidance in clinical practice.

Cooperation between member states in the area of HTA has increased considerably over the last decade. This is reflected amongst other things in alliances such as EUnetHTA, a collaboration between European HTA organisations aiming to facilitate efficient use of resources available for HTA in order to create a sustainable system of HTA knowledge sharing and to promote good practice in HTA methods and processes [17]. By means of providing guidance on the use of appropriate methodologies and capacities with respect to molecular diagnostics, EUnetHTA may help to support timely access for patients to these technologies across Europe.

\section{Rewarding the Value of Molecular Diagnostics}

\section{Current Willingness to Pay for Molecular Diagnostics}

It is estimated that diagnostics account for $<2 \%$ of total health care spending but influence $60 \%$ of clinical decision-making [18]. In both the US and the EU, molecular diagnostics are generally reimbursed under a cost-based, administered pricing system. As discussed in the previous section, the assessment of the value of these tests remains to be clarified in most health care systems. The current approach does not reward value creation, nor does it incentivise evidence generation to support the demonstration of value. As a consequence, most European patients are effectively denied access [4]. Innovative molecular diagnostics provide medical and economic value to patients, their physicians, and health care payers, and this should be reflected in the pricing. In most of the European health care systems, the willingness to pay for diagnostics priced according to their value remains unclear and often seems to be quite limited [19]. Payers expect diagnostics to be cost neutral at best, and they do not seem to fully appreciate the value that these technologies may bring to clinical decision-making.

Molecular diagnostic tests have been available in $\mathrm{Eu}-$ rope for a number of years, with the vast majority being utilised in oncology $[19,20]$. However, there is widespread recognition that a fragmented commissioning system for these tests has led to varied access, with patients missing out on appropriate treatment options [20]. Indeed, in the case of medicines, positive reimbursement recommendations normally lead to a clear commissioning route with dedicated budget allocation. However, in the case of (molecular) diagnostics, positive assessment leading to a reimbursement recommendation does not necessarily come with clarity on the budget source, nor is a dedicated budget allocation identified. In Germany and the UK, it is expected that hospitals initially fund new diagnostic tests out of existing hospital tariffs [diagnosisrelated groups (DRGs)], and that these DRGs will then be increased after a period of time (e.g. when cost savings in other DRGs occur as the result of molecular testing). This system limits the integration of new tests into clinical practice, but it also does not incentivise manufacturers to generate high-quality evidence to support the value of their new test $[11,12]$. The overall effect of these limitations to the reimbursement system is that patients have limited and unequal access to those new tests (fig. 1). If these reimbursement systems were fit for purpose, diagnostics-enabled health care would be more cost-effective. Moreover, manufacturers would be more inclined to invest in research to develop new tests and generate evidence to demonstrate the clinical and economic value of these tests.

Attention must be devoted to the issue of budget silos: it is not uncommon in health care for savings to occur in one budget but the innovation to be paid for from a different budget [3]. This can lead to perverse incentives where the chance of a new health technology reaching patients becomes even less likely [21]. Moreover, the tariff payment system may lead to counterincentives for hospitals. This is the case for predictive molecular tests such as the Oncotype $D X^{\circledR}$ test for early-stage breast cancer [22]. This test has the potential to pay off by reducing chemotherapy use and costs, but because the total chemotherapy cost (drugs, management of side effects, etc.) is borne by different parties/budgets within the health care system, no one party has a clear incentive to fund the test to recoup the savings. With chemotherapy attached to specific tariff payment from commissioners, hospitals may lose revenue by receiving fewer chemotherapy tariffs and be penalised from a financial perspective. Again, better alignment between financial incentives and the clinical value of new molecular tests is needed to allow more equitable access for patients to these new tests. 
Fig. 1. Effects of limitations to reimbursement systems. Budget silos and counterincentives of tariff payment systems.

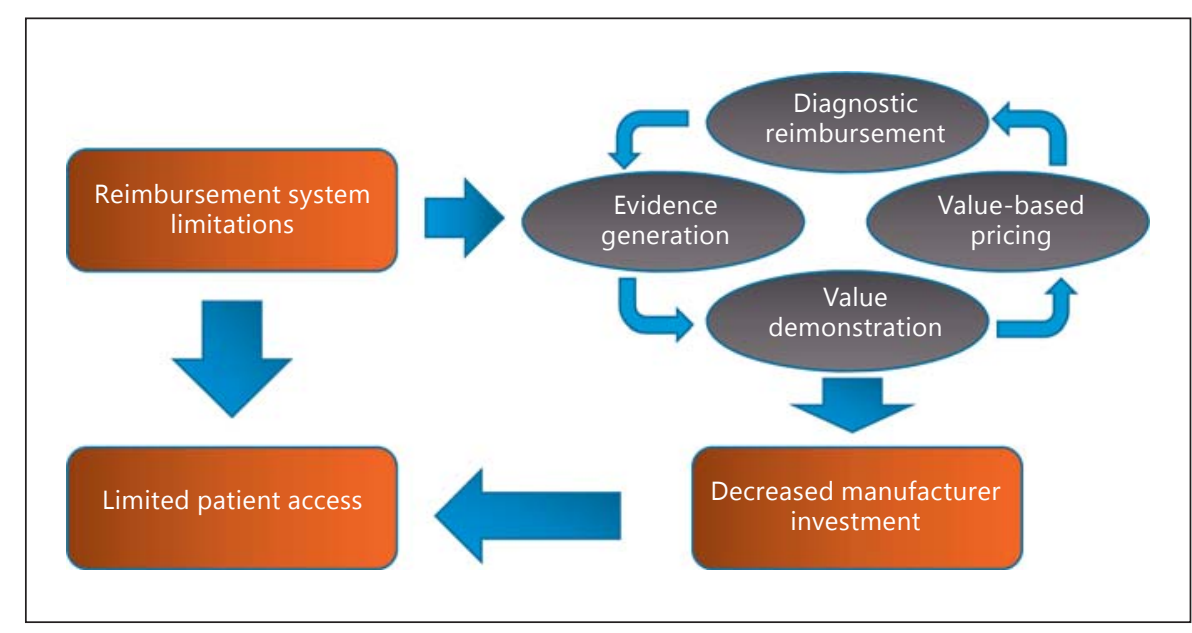

\section{Case Studies}

\section{Oncotype DX ${ }^{\circledR}$ Breast Cancer Assay}

The Oncotype $\mathrm{DX}^{\circledR}$ breast cancer test is a 21 -gene molecular diagnostic test which is used in early-stage breast cancer to inform chemotherapy decisions following surgery. This test has been shown to be prognostic (prediction of breast cancer recurrence at 10 years) and predictive of chemotherapy benefit [23]. Its impact on treatment decisions in clinical studies [22] as well as real-life settings $[24,25]$ is well documented, leading to a significant reduction of the use of chemotherapy. There is also a wealth of evidence supporting the costeffectiveness of this test versus current clinical practice from a variety of countries [26]. More recently, large prospective studies have confirmed the prospective-retrospective studies that had initially supported the clinical validation of this test, confirming that withdrawing chemotherapy in patients that had a low recurrence score on the Oncotype $\mathrm{DX}^{\circledR}$ test led to excellent patient outcomes [27-30]. Despite its inclusion in international and European clinical guidelines [31-34], and its recommendation by NICE [35], its reimbursement remains quite limited in Europe. Genomic Health was one of the first diagnostic companies to implement valuebased pricing, and this proved to be very challenging for all the reasons explained above. Also, Genomic Health's central laboratory model, which presents many advantages in terms of the quality and reproducibility of the test, is being challenged by some of the health authorities. Indeed, the transfer of value to the US is not always well accepted. Some countries, however, made the decision to reimburse this test early, before NICE issued its

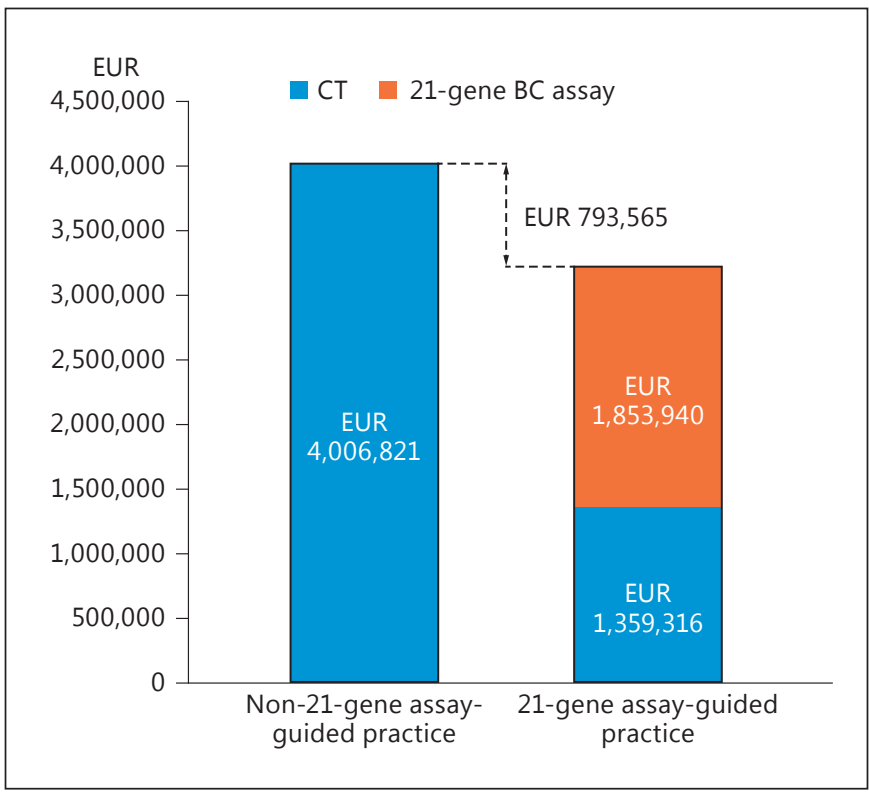

Fig. 2. Economic impact of the use of the Oncotype $D X^{\circledR}$ test in Ireland.

guidance and before the publication of prospective evidence. Ireland was the first European country to reimburse the Oncotype DX ${ }^{\circledR}$ test in October 2011. When the TAILORx trial ended its recruitment in Ireland in 2009, the National Cancer Control Programme (NCCP) asked the National Centre for Pharmacoeconomics (NCPE) to assess the Oncotype DX ${ }^{\circledR}$ test. The HTA and the pricing negotiations were completed within 9 months, and the positive reimbursement decision proved to be cost-effective as it led to significant savings 
for the Irish Department of Health, as shown in figure 2 [25]. This positive experience was even shared in a conference that took place in 2013 under the auspices of the Irish EU presidency [3].

The province of Ontario in Canada decided to reimburse the Oncotype DX ${ }^{\circledR}$ test in 2010 following an HTA conducted by the Ontario Health Technology Advisory Committee (OHTAC) [36]. Because the HTA report raised some uncertainties related to the expected impact of the test in real-life clinical practice, the province of Ontario decided to conduct a field evaluation in order to address this question and to revisit the cost-effectiveness evaluation based on the findings from the field evaluation. The results from this study showed that the test led to a $53 \%$ change in treatment decisions (with a $38 \%$ reduction in the use of chemotherapy) [24], and the costeffectiveness analysis [37] indicated that using the test was cost-effective. These results confirmed the original decision made by the province of Ontario, and since then patients continue to have access to the Oncotype DX ${ }^{\circledR}$ test.

\section{KRAS Companion Diagnostic}

CRC is one of the most common cancers worldwide. With the emergence of two anti-epidermal growth factor receptor (EGFR)-targeted antibodies, cetuximab (Erbitux) and panitumumab (Vectibix), the treatment of metastatic CRC has entered into the era of personalised treatment. However, EGFR, the target of these drugs, which is overexpressed in approximately $80 \%$ of colorectal carcinomas, failed to predict a therapeutic response when used clinically. The KRAS gene has been demonstrated to be a strong negative predictive biomarker to indicate whether a CRC patient will respond to anti-EGFR treatment. As the target treatment may also be toxic and expensive, KRAS mutation status detection has become a crucial diagnostic factor for treating metastatic CRC patients [38]. A decision of the French public health insurance system to provide targeted therapy for CRC, together with a joint initiative between the National Cancer Institute (INCa) and the French Ministry of Health, has had a positive impact on access to KRAS testing in France, as discussed in Box 3. The French screening and targeted cancer treatment initiative offers equal access to molecular testing for all patients in France and represents a real benefit in terms of public health. It illustrates that molecular stratification can be successfully integrated into the health care system and, as an additional benefit, is a cost-effective strategy $[39,40]$.

Equal Access to Molecular Diagnostics

\section{Box 3}

In order to ensure wide access to molecular tumour profiling, INCa and the Ministry of Health set up a national network of 28 regional molecular genetics centres in 2006, aiming at uniform nationwide test provision and fast implementation of molecular tests for new tumour biomarkers, including KRAS mutation testing. With the aim of serving as a regional hub for expert molecular testing, each molecular genetic centre is based on a partnership between several university hospital and cancer centre laboratories with complementary expertise. Each laboratory developed KRAS testing according to its own expertise and to the locally available technology platform $[39,40]$. INCa is responsible for coordinating the 28 regional molecular genetics centres at the national level; the network is funded by INCa and the Ministry of Health. During the setup phase, INCa allocated EUR 4.7 million for the purchase of equipment; this initial funding was followed by the allocation of EUR 4 million in annual funding to the centres and staff from the French Ministry of Health. Additional, specific funding was required following the authorisation of panitumumab and cetuximab for patients with wild-type KRAS, because a sharp increase in molecular testing activity was expected. INCa allocated an additional EUR 2.5 million for KRAS mutation screening to the 2008 budget. INCa used quarterly reports to monitor the activity of the centres and to adjust the overall budget and its allocations accordingly. This additional funding was then followed by recurrent annual funding from the French Ministry of Health [40]. A sharp increase in KRAS and EGFR mutation screening of colorectal and lung cancers was seen after antiEGFR and EGFR tyrosine kinase inhibitor therapy approvals. In 2009, 17,250 patients benefited from KRAS mutation screening, compared to only 1,100 in 2007. The activity has stabilised as expected since 2010 , with KRAS testing of 16,581 patients in 2010 and 17,003 patients in 2011. A range of studies have indicated the cost-effectiveness of KRAS testing. For example, KRAS testing avoids a median of 8 months of ineffective cetuximab therapy in patients with KRAS mutations that would cost EUR 32,419 per patient in France [40].

\section{Policy and HTA Implications for More Equitable Access to Molecular Diagnostic Tests in Europe}

\section{Greater Involvement of Patients}

To realise the potential of molecular diagnostics in Europe, access to these technologies urgently needs to be improved. In order to achieve this, funding and reimbursement systems need to be adapted and move from a 'fee per service' towards a 'value-based' approach, rewarding patient outcomes. Achieving the best results for patients should be the highest priority. The voice of patients should therefore be represented in HTA and reimbursement processes and reflected in ultimate decisions, in particular at a time when new (disruptive) technologies 
offer important opportunities for patients. The representation of patients in reimbursement processes may still be considered limited [41].

In addition, in most HTA and reimbursement processes in Europe, patients are not directly represented. Apart from financial resource constraints, the main challenges regarding patients' involvement are perceived to be the lack of capacity, time, and good methodologies [14]. Yet, patients have valuable perspectives and experience that can inform HTA and decision-making, helping to explain what it is like to live with a particular condition and shedding light on their experience with current technologies and what they would value most in a new treatment. In the appraisal phase of HTA, patient evidence can inform discussions about the added value of a new technology and generally inform the value judgements of researchers and decision-making committees. Active involvement of patients will result in technology assessments of higher quality compared to those currently performed, maximising the potential for a technology's adoption and implementation [2]. There are initiatives at the EU level aiming to ensure patients' interests are represented in a meaningful way in HTA and reimbursement discussions. These are driven by European patient organisations (such as the European Patients' Forum [14] and EURORDIS Rare Diseases Europe [42]) and by projects such as ADAPT SMART [43], which seeks to establish collaborative solutions to foster the development of Medicines Adaptive Pathways to Patients (MAPPs) [44], aiming to provide patients with more appropriate access to innovative medicines. The European Patients' Academy on Therapeutic Innovation (EUPATI) project on the other hand focuses on capacity building to enhance patients' knowledge and enable them to engage effectively in therapeutic R\&D [45]. These initiatives focus mostly on medicinal products, but they could potentially be extended to include medical/diagnostic technologies.

\section{Clearer Reimbursement Pathways Including Evidence Requirements}

Reimbursement of molecular diagnostic tests in Europe is challenging for a variety of reasons. Most importantly, only few reimbursement or uptake decisions are made based on value - reimbursement is generally based on lump sums for health care institutions and laboratories, with cost and price being the only criteria. A dedicated and specific value-based reimbursement pathway for innovative (molecular) diagnostic technologies needs to be developed and implemented consistently across Europe, ensuring equal access for patients. In order for these pathways to be effective, it is essential that clear evidence requirements are set. Different health care systems in Europe ask for different and increasing evidence of the value that diagnostic technologies contribute. Decision-makers, including HTA bodies, informed by input from patients, should define the key value outcomes they seek from diagnostic technologies and should commit to pay for them [46].

\section{Better Implementation of HTA Guidance}

Once HTA processes have been adapted to include the interests of patients and appropriate methods and skills to evaluate innovative molecular diagnostics are in place, there is a need to ensure that the resulting guidance is actually implemented. In many countries, HTA processes do not automatically lead to funding or reimbursement decisions [16]. It is therefore key to ensure that published HTA guidance results, if positive, lead to a clear commissioning route with a dedicated budget. This can be achieved through better alignment of the HTA and the commissioning process and by making the HTA guidance legally binding, at the same time monitoring its implementation into clinical practice.

\section{Coverage with Evidence Development}

Coverage with evidence development may offer opportunities for earlier patient access to innovative molecular diagnostic tests. Indeed, conditional/temporary reimbursement of new technologies against data collection from providers could be a solution to facilitate rapid and safe patient access. Coverage with evidence development is usually implemented when there is either a need to manage utilisation in real-world situations or to provide evidence in order to address the uncertainty of the reimbursement decision [47]. Assuming that the process of data collection being requested against reimbursement is not too cumbersome for providers, this may be a solution for earlier access for patients that also satisfies payers' and manufacturers' expectations. As presented in the Oncotype $\mathrm{DX}^{\circledR}$ case study earlier, conditional reimbursement was successfully implemented by the province of Ontario in Canada. This positive experience could be replicated in other countries.

\section{Conclusions}

In the area of molecular diagnostics, further reform is needed to ensure patients can realise benefits from these tests and receive equal access leading to the best possible 
clinical outcomes. Clear reimbursement pathways and evidence requirements, together with a systematic implementation of reimbursement recommendations following HTA, are needed. Moreover, conditional reimbursement of further data collection may be a solution to allow earlier access for patients while enabling payers to receive the answers to some of the key questions they may have on the real-life impact of (molecular) diagnos- tics. Finally, it is key that patients are involved more in value assessment via HTA as well as in reimbursement discussions.

\section{Disclosure Statement}

The authors confirm that they do not have any conflicts of interest.

\section{References}

1 Council Conclusions on Personalised Medicine for Patients, adopted 7 December 2015. http://data.consilium.europa.eu/doc/document/ST-14393-2015-INIT/en/pdf.

2 European Alliance for Personalised Medicine: Affordable and sustainable patient access to personalised medicine. http://euapm. eu/pdf/EAPM_Affordable_and_sustainable patient_access_to_PM.pdf.

3 European Alliance for Personalised Medicine: Report from Irish Presidency Conference March 20th/21st 2013. http://euapm.eu/ pdf/EAPM_REPORT_on_Innovation_and Patient_Access_to_Personalised_Medicine. pdf.

4 Miller I, et al: Market access challenges in the EU for high medical value diagnostic tests. Pers Med 2011;8:137-148.

5 Monzon FA, et al: The role of KRAS mutation testing in the management of patients with metastatic colorectal cancer. Arch Pathol Lab Med 2009;133:1600-1606.

6 Health for all, care for you: primary results of the Science|Business and Karolinska Institutet survey on the promise of personalised healthcare in Europe. 2010. http://www.sciencebusiness.net/pdfs/PM_survey_results. pdf.

7 European Alliance for Personalised Medicine: Better treatment through better education: a European education strategy for the PM area. http://euapm.eu/pdf/EAPM_Better_ treatment_through_better_education_A_ European_education_strategy_for_the_PM era.pdf.

8 Faulkner E, et al: Challenges in the development and reimbursement of personalised medicine - payer and manufacturer perspectives and implications for health economics and outcomes research: a report of the ISPOR personalized medicine special interest group. Value Health 2012;15:1162-1172.

9 International Society for Pharmacoeconomics and Outcomes Research: Germany medical devices. 2011. http://www.ispor.org/ HTARoadMaps/GermanyMD.asp.

10 International Society for Pharmacoeconomics and Outcomes Research: France medical devices. 2011. http://www.ispor.org/ HTARoadMaps/FranceMD.asp.
11 Garau M, et al: Can and should value-based pricing be applied to molecular diagnostics? Pers Med 2013;10:61-72.

12 Garrison L: The economics of personalized medicine: a model of incentives for value creation and capture. Drug Inform J 2007;41: 501-509.

13 Garrison L, Towse A: Economics of personalized medicine: pricing and reimbursement policies as a potential barrier to development and adoption; in Culyer AJ (ed): Encyclopedia of Health Economics. Amsterdam, Elsevier, 2014, pp 484-490.

14 European Patients' Forum: EU patient involvement in Health Technology Assessment (HTA). http://www.eu-patient.eu/whatwedo/ Initiatives/.

15 Garrison L, et al: The value of knowing and knowing the value: improving the health technology assessment of complementary diagnostics. In press.

16 National Institute for Health and Care Excellence: NICE diagnostics guidance. https:// www.nice.org.uk/about/what-we-do/ourprogrammes/nice-guidance/nice-diagnostics-guidance.

17 EUnetHTA: About us. http://www.eunethta. eu/about-us.

18 Gustavsen G, Phillips K, Pothier K: The reimbursement landscape for novel diagnostics. 2010. https://www.bio.org/sites/default/files/ Health_Advances\&BIO_Novel_Diagnostics_Reimburs_20110103_0.pdf.

19 Garfield S: Advancing access to personalized medicine: a comparative assessment of European reimbursement systems. http://www. personalizedmedicinecoalition.org/Userfiles/PMC-Corporate/file/pmc_bridgehead_ issue_brief_european_reimbursement.pdf.

20 British in vitro Diagnostics Association: Robust, fair and sustainable: our vision for the future access and provision of molecular diagnostics in England. 2015. http://www.bivda.co.uk/Portals/0/Documents/Policy\%20 and\%20Media/BIVDA\%20Vision\%20document.pdf.
21 Katz G, et al: Economic impact of gene expression profiling in patients with early-stage breast cancer in France. PLoS ONE 2015; 10:e0128880

22 Augustovski F, et al: Decision-making impact on adjuvant chemotherapy allocation in early node-negative breast cancer with a 21-gene assay: systematic review and meta-analysis. Breast Cancer Res Treat 2015;152:611-625.

23 Markopoulos C: Overview of the use of Oncotype DX ${ }^{\circledR}$ as an additional treatment decision tool in early breast cancer. Expert Rev Anticancer Ther 2013;13:179-194.

24 Levine MN, et al: Prospective evaluation of the 21-gene recurrence score assay for breast cancer decision-making in Ontario. J Clin Oncol 2016;34:1065-1071.

25 Smyth L, et al: Economic impact of 21-gene recurrence score testing on early-stage breast cancer in Ireland. Breast Cancer Res Treat 2015; 153:573-582.

26 Rouzier R, et al: Multigene assays and molecular markers in breast cancer: systematic review of health economic analyses. Breast Cancer Res Treat 2013;139:621-637.

27 Sparano JA, et al: Prospective validation of a 21-gene expression assay in breast cancer. N Engl J Med 2015;373:2005-2014.

28 Shak S, et al: Breast cancer specific survival in 38,568 patients with node negative hormone receptor positive invasive breast cancer and Oncotype DX recurrence score results in the SEER database. 38th Ann CTRC-AACR San Antonio Breast Cancer Symposium, December 8-12, 2015, San Antonio. Cancer Res 2016;76:P5-15-01.

29 Stemmer SM, et al: Real life analysis evaluating 2,028 No/N1mic breast cancer patients for whom treatment decisions incorporated the 21-gene recurrence score results: 5 years KM estimate for breast cancer specific survival below $<30$ is $>98 \%$. 38th Ann CTRC-AACR San Antonio Breast Cancer Symposium, December 8-12, 2015, San Antonio.

30 Gluz O, et al: West German Study Group Phase III PlanB Trial: first prospective outcome data for the 21-gene recurrence score assay and concordance of prognostic markers by central and local pathology assessment. J Clin Oncol 2016, Epub ahead of print. 
31 Harris LN, et al: Use of biomarkers to guide decisions on adjuvant systemic therapy for women with early-stage invasive breast cancer: American Society of Clinical Oncology Clinical Practice Guideline. J Clin Oncol 2016;34:1134-1150.

32 NCCN Clinical Practice Guidelines in Oncology $^{\mathrm{TM}}$ Breast Cancer (version 1.2011). http:// www.nccn.org.

33 Coates AS, et al: Tailoring therapies - improving the management of early breast cancer: $\mathrm{St}$ Gallen International Expert Consensus on the Primary Therapy of Early Breast Cancer 2015. Ann Oncol 2015;26:1533-1546.

34 Aebi S, et al: Primary breast cancer: ESMO Clinical Practice Guidelines for diagnosis, treatment and follow-up. Ann Oncol 2010; 21(suppl 5):v9-14.

35 National Institute for Health and Care Excellence: Guidance. 2013. https://www.nice.org. uk/Guidance/DG10.
36 Ontario Health Technology Advisory Committee: OHTAC recommendation. 2010. http://www.hqontario.ca/english/providers/ program/ohtac/tech/recommend/rec gep_20101213.pdf.

37 Paulden M, et al: Cost-effectiveness of the 21gene assay for guiding adjuvant chemotherapy decisions in early breast cancer. Value Health 2013;16:729-739.

38 Tan C, Du X: KRAS mutation testing in metastatic colorectal cancer. World J Gastroenterol 2012;18:5171-5180.

39 Blons $\mathrm{H}$, et al: Performance and cost efficiency of KRAS mutation testing for metastatic colorectal cancer in routine diagnosis: the MOKAECM study, a nationwide experience. PLoS One 2013;8:e68945.

40 Nowak F, et al: Tumour molecular profiling for deciding therapy - the French initiative. Nat Rev Clin Oncol 2012;9:479-486.

41 Noordman J, et al: Patient organisations and the reimbursement process for medicines: an exploratory study in eight European countries. BMC Health Serv Res 2010;10:45.
42 EURORDIS: Health technology assessment. 2013. http://www.eurordis.org/content/healthtechnology-assessment- 0 .

43 ADAPTSMART. http://adaptsmart.eu.

44 European Medicines Agency: Adaptive pathways. http://www.ema.europa.eu/ema/index. jsp?curl=pages/regulation/general/general_ conten t_000601.jsp\& m id = WC0b01ac05807d58ce.

45 European Patients' Academy. http://www. eupati.eu.

46 Wurcel V: The value of diagnostic information and why it matters. MedTech Views, December 3, 2015. http://medtechviews.eu/ article/value-diagnostic-information-andwhy-it-matters.

47 Towse A: Performance-based risk-sharing arrangements: good practices for design, implementation and evaluation. ISPOR 2012 http://www.ispor.org/meetings/WashingtonDC0512/releasedpresentations/FINALAdrian-Towse.pdf. 